\title{
Polydactyly of a biphalangeal thumb
}

INSERM

\section{Source}

INSERM. (1999). Orphanet: an online rare disease and orphan drug data base. Polydactyly of a biphalangeal thumb. ORPHA:93339

Polydactyly of a biphalangeal thumb or PPD1 is the most common form of preaxial polydactyly of fingers (see this term), a limb malformation syndrome, that is characterized by the duplication of one or more skeletal components of a biphalangeal thumb. Hands are preferentially affected (in bilateral), and the right hand is more commonly involved than the left. 